\title{
Development of a Turbulent Cascade behind the Bow Shock under Quiet Conditions in the Solar Wind
}

\author{
L. S. Rakhmanova ${ }^{a, *}$, M. O. Riazantseva ${ }^{a, * *}$, G. N. Zastenker ${ }^{a}$, and Yu. I. Yermolaev ${ }^{a}$ \\ a Space Research Institute, Russian Academy of Sciences, Moscow, 117997 Russia \\ *e-mail: rakhlud@gmail.com \\ **e-mail: orearm@gmail.com
}

Received March 11, 2021; revised March 29, 2021; accepted May 27, 2021

\begin{abstract}
The bow shock crossing by the solar wind can lead in a number of cases to significant changes in the development of the turbulent cascade. Individual cases previously studied on the basis of experimental measurements of the characteristics of turbulence in the magnetosheath have not yet identified the factors that have the greatest influence on the modification of the turbulent cascade behind the bow shock. In this paper, we consider several observation cases of spectra of the compressible component in magnetosheath fluctuations on two satellites separated in space under calm conditions in the solar wind. This makes it possible to estimate the influence of the magnetosheath boundaries and the bow shock topology on the dynamics of a turbulent cascade when the plasma moves behind the bow shock. It is shown that there is a significant redistribution of energy in the turbulent cascade immediately behind the quasi perpendicular bow shock in the daytime part of the magnetosheath. This affects the magnetohydrodynamic scales, and the cascade properties are restored upon further propagation of the plasma towards the flanks. At the same time, behind the quasi parallel bow shock, the characteristics of the turbulent cascade upon the entry of plasma into the magnetosheath change only on subionic scales.
\end{abstract}

DOI: $10.1134 / \mathrm{S} 0016793221050108$

\section{INTRODUCTION}

The interaction of the solar wind (SW) with the Earth's magnetosphere leads to the formation of the bow shock (BS). The region between the BS and the magnetopause, the magnetosheath (MSH), is characterized, among other things, by a higher level of plasma compression, the development of various wave processes and, as a consequence, higher power density of fluctuations in comparison with the undisturbed SW upstream the BS. An important feature of cosmic plasma, which is also characteristic of the MSH and SW, is its turbulence. The turbulent cascade in the SW has been studied since the launch of the first satellites (see reviews by Alexandrova et al., 2013; Bruno and Carbone, 2013), while active research on turbulence in the MSH began relatively recently (e.g., reviews by Sahraoui et al., 2020; Rakhmanova et al., 2021)). A significant part of studies on turbulence in SW and MSH in recent decades has attempted to determine the properties of a turbulent cascade on scales of the order of the proton gyroradius or less, at which kinetic processes play an important role and plasma heating is believed to occur.

Since the characteristics of the SW and interplanetary magnetic field (IMF) plasma are significantly modified when they cross the BS, the properties of the turbulent cascade can also change. Statistical analysis of the characteristics of plasma turbulence in the MSH on scales near the proton gyroradius (i.e., when one passes from MHD to a kinetic description of the plasma) showed their difference from observations in an undisturbed SW, as well as the dynamics of turbulence characteristics during plasma propagation in the MSH from the BS to the flanks (e.g., Huang et al., 2017; Rakhmanova et al., 2018; Rakhmanova et al., 2018; Li et al., 2020). In particular, according to statistical studies of magnetic-field fluctuations measured on satellites of the Cluster mission (Huang et al., 2017) and ion-flux fluctuations measured on the Spektr-R satellite (Rakhmanova et al., 2018; Rakhmanova et al., 2018), it was shown that spectra observed in the subsolar region in the MSH near the BS on MHD scales as a rule can be approximated by a power function $\sim f^{-1}$ (where $f$ is the frequency of the fluctuations measured in the plasma reference frame) instead of the function $f^{-5 / 3}$, which is characteristic of the developed Kolmogorov turbulence in undisturbed SW. In this case, this effect becomes less pronounced with distance from the BS in the direction of the magnetopause and in the direction of the flanks. Differences in the shapes of the spectra typical for plasma near the BS and in the inner regions of the MSH were shown on smaller (subionic) scales, which may indicate a difference in the processes of dissipation of plasma energy. 
However, an experimental study in recent years of individual events (Rakhmanova et al., 2019; Rakhmanova et al., 2020), as well as a comparison of observations on various spacecraft presented in the works of different authors (Rakhmanova et al., 2021), revealed different scenarios for the modification of a turbulent cascade on the BS. The considered cases were observed under different conditions in the SW and different positions of satellites behind the BS. It was suggested that the characteristics of the turbulent cascade behind the BS and its development during plasma propagation in the MSH can be influenced by such factors as the type of large-scale SW flow, the mutual orientation of the IMF and normal to theBS, and the distance from the point of plasma entry into MSH to the subsolar point of theBS. However, the degree, method, and conditions of the influence of each of these factors have not yet been determined.

In this work, we analyze the dynamics of the characteristics of plasma turbulence behind the BS under quiet conditions in the SW based on four cases of the simultaneous observations on two satellites, the Spektr-R spacecraft and one of the satellites of the Themis mission, of the frequency spectra of fluctuations inside the MSH. The fixing of one important factors, the type of the SW incoming flow, made it possible to analyze in isolation the influence of the BS topology and the distance to it on the development of a turbulent cascade inside the MSH.

\section{DATA AND METHODS OF ANALYSIS}

In this work, we used measurements of the ion flux on the Spektr-R satellite by the BMSW instrument (Zastenker et al., 2013; Šafránková et al., 2013) with a time resolution of $0.031 \mathrm{~s}$ and the magnetic-field magnitude on satellites of the Themis mission with FGM instruments (Auster et al., 2008) with a time resolution of $0.25 \mathrm{~s}$. The high temporal resolution of measurements makes it possible to analyze fluctuations of parameters in the frequency range corresponding to the transition from MHD to kinetic scales. In addition, plasma measurements from electrostatic analyzers (ESAs) on Themis satellites with a temporal resolution of $4 \mathrm{~s}$ were used to make comparisons between a pair of satellites (McFadden et al., 2008). In the selected time periods, the satellites were located on the same flank of the MSH or at a small distance (less than $20 R_{\mathrm{E}}$ ) in the subsolar region. At the same time, the conditions in the incoming SW flow were checked: only events in slow, undisturbed SW flow were selected-type Slow according to the catalog of Yermolaev et al. (2009). The catalog is available on the Internet (http://www.iki.rssi.ru/pub/omni/catalog). For the analysis, we selected intervals with a duration of at least 20 min without large amplitude changes in the plasma parameters and/or the magnetic-field magnitude, nor rotations of the magnetic-field vector, which may indicate the intersection of plasma structures.
In the first stage, the ion flux time course measured on two satellites was compared. Figure 1 shows the ion flux observed on October 30, 2011, by the Themis-A (black line, left ordinate axis) and Spektr-R (gray line, right ordinate axis) satellites. Figure $2 a$ shows the relative position of the satellites. Measurements of the Themis-A satellite were shifted by the time of plasma propagation (10 $\mathrm{min})$. Themis-A was near the subsolar part of the magnetopause; it crossed it at $\sim 0135$ UT and entered the magnetosphere. Spektr-R was on the flank, at a distance of $\sim 25 R_{\mathrm{E}}$ along the axis $X_{G S E}$ (geocentric solar ecliptic system, $G S E$ ), away from both MSH boundaries. The angle $\theta_{B N}$ between the local normal to the BS at the point of plasma entry into the MSH and the IMF for both satellites was $60^{\circ}$. Angle $\theta_{B N}$ was calculated at the point of plasma entry into the MSH, which was determined via satellite tracing to the BS along the streamlines calculated with the Spreiter model (Spreiter et al., 1966). The technique was described in detail by Shevyrev and Zastenker (2005). Thus, both satellites were located behind the quasi perpendicular BS but at different distances from the subsolar point. A good visual correspondence is observed with the time course of the ion flux on both satellites, which indicates that the same plasma volume is observed on them. Vertical dashed lines indicate a time interval of about an hour, which was used for further analysis.

Fourier analysis was used to determine the characteristics of the turbulence. In this case, the fluctuations of the parameters were normalized to the average value of the corresponding parameter over the entire interval prior to the calculation of the frequency spectra. The indicated normalization was carried out to compare the power of fluctuations of various parameters. It should be noted that both the ion-flux fluctuations and the fluctuations in the magnetic-field magnitude are compression fluctuations (e.g., Alexandrova et al., 2008); accordingly, comparison of the characteristics of their frequency spectra is physically justified. The possibility of comparing the spectra of fluctuations of the ion flux and the magnetic field magnitude was considered in more detail (Rakhmanova et al., 2020), and a direct comparison of the spectra of different quantities on the same spacecraft has been given in the literature (Chen, 2016; Chen and Boldyrev, 2017; Roberts et al., 2017). Figure $2 b$ shows the spectra of fluctuations of the ion flux on the Spektr-R satellite (gray line) and the magnetic-field magnitude on the Themis-A satellite (black line). Both spectra can be approximated by two power law functions changing each other at a certain frequency (break frequency). The exponents of power law functions (the so-called spectral slope) were determined on the MHD and kinetic scales based on a linear approximation (at frequencies below and above the break, respectively). The spectrum of fluctuations of the magnetic-field magnitude observed by the Themis-A satellite is characterized by a slope of $-1.09 \pm 0.08$ on the MHD scales 


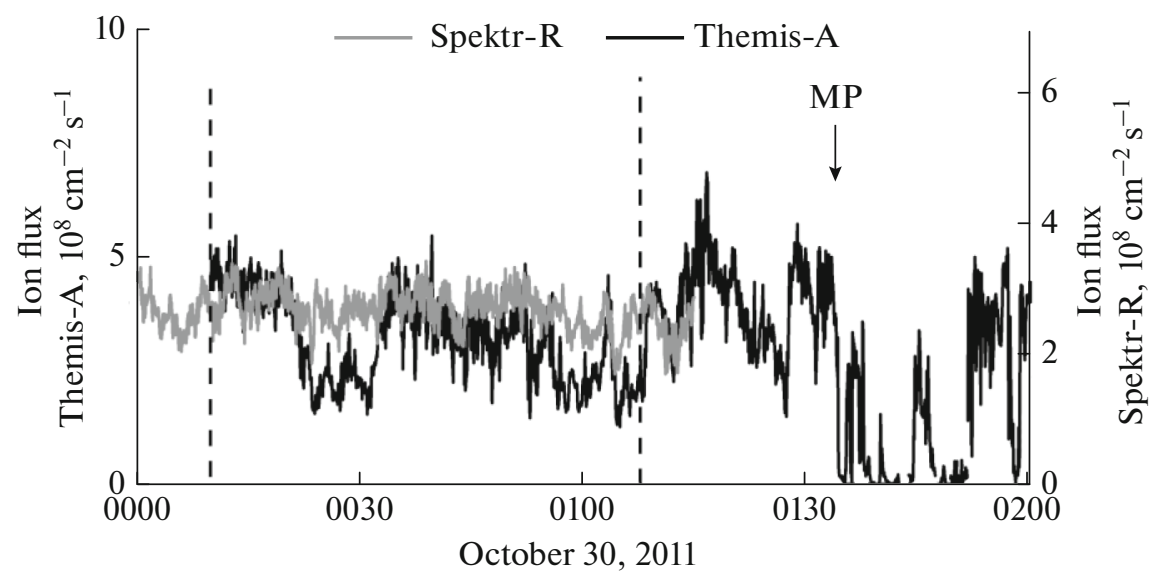

Fig. 1. Time variation of the ion flux in the magnetosheath measured on the Themis-A satellite (black line, left ordinate axis) and Spektr-R satellite (gray line, right ordinate axis). The vertical dashed lines indicate the boundaries of the interval selected for spectral analysis.

and $-2.88 \pm 0.01$ on the kinetic scales. The spectrum of ion-flux fluctuations observed downstream by the Spektr-R satellite is characterized by a slope of $-1.50 \pm$ 0.02 on the MHD and $-2.97 \pm 0.01$ on the kinetic scales, respectively. It is clearly seen that the properties of the turbulent cascade on MHD scales in the subsolar region are very different from the predictions of the theory of developed turbulence, i.e., they do not follow the Kolmogorov scaling, despite the satellite's remoteness from the BS. On the kinetic scales, the slope of the spectrum is close to the typical value -(2.7-2.9) observed on average in statistical studies of turbulence in the MSH (Huang et al., 2014; Rakhmanova et al., 2016), as well as in SW (Chen et al., 2012; Riazantseva et al., 2015), and is close to some theoretical predictions (e.g., Boldyrev and Perez, 2012). As the plasma moves away from the subsolar region, the slope of the spectrum on the MHD scales approaches the Kolmogorov scaling, while a slight steepening of the kinetic part of the spectrum is observed. As a rule, it is believed that the frequency of the break in the spectrum is associated with the dominant process of energy dissipation and corresponds to a certain characteristic plasma frequency. However, it has not yet been possible to determine unambiguously the frequency that corresponds to the break(e.g., Šafránková et al., 2016). Determining the position of the break frequency is beyond the scope of this work; however, the characteristic frequencies are given for reference for each spectrum in Figure 2 along the abscissa axis. The dotted line denotes the proton gyrofrequency $F_{C}$. The frequencies $F_{G}=V / 2 \pi \rho$ and $F_{L}=V / 2 \pi L$ (where $V$ is the plasma flow velocity), which are determined by the Larmor radius $\rho$ and the proton inertial length $L$, are indicated by the dashed and solid lines, respectively. The frequencies for the Themis satellite are black, and the frequencies for the Spektr-R satellite are gray. Since there are no magnetic-field measurements on the Spektr-R satellite, only the last of the listed frequencies is given for it.

\section{RESULTS}

Three more events with different BS topologies and with different spacecraft localizations inside the MSH were analyzed in a similar way. In this case, the visual correspondence of the ion flux time course on two satellites was checked for each event in order to exclude the registration of fundamentally different plasma volumes. Figures $2 \mathrm{c}, 2 \mathrm{e}$, and $2 \mathrm{~g}$ show the locations of pairs of satellites during the considered events; Figures 2d, 2f, and 2h compare the spectra of fluctuations of parameters in the indicated events, respectively. The black lines indicate the spectra of fluctuations of the magnetic-field magnitude from Themis satellite measurements, and the gray lines show the spectra of ion-flux fluctuations by measurements of the Spektr-R satellite. Angle $\theta_{B N}$, which characterizes the BS type, is indicated on the panels on the left with the location for each spacecraft. The results of approximation of the spectral slopes on the MHD and kinetic scales are shown in the panels on the right next to the corresponding curve. Table 1 gives the features of the satellite locations for each event, as well as the characteristics of the fluctuation spectra. In the column "Location," the letters "f" and "s/s" mark the flank and subsolar regions, respectively, "Center" denotes the distance from the boundaries of the MSH; "MP" and "BS" denote the proximity to the magnetopause or BS, respectively. In this case, the location near the boundaries is understood as a situation in which its intersection occurs within $1 \mathrm{~h}$ before or after the analyzed interval; otherwise it refers the distance of the satellite from the boundaries.

Comparison of the characteristics of the spectra of parameter fluctuations for four events with different 

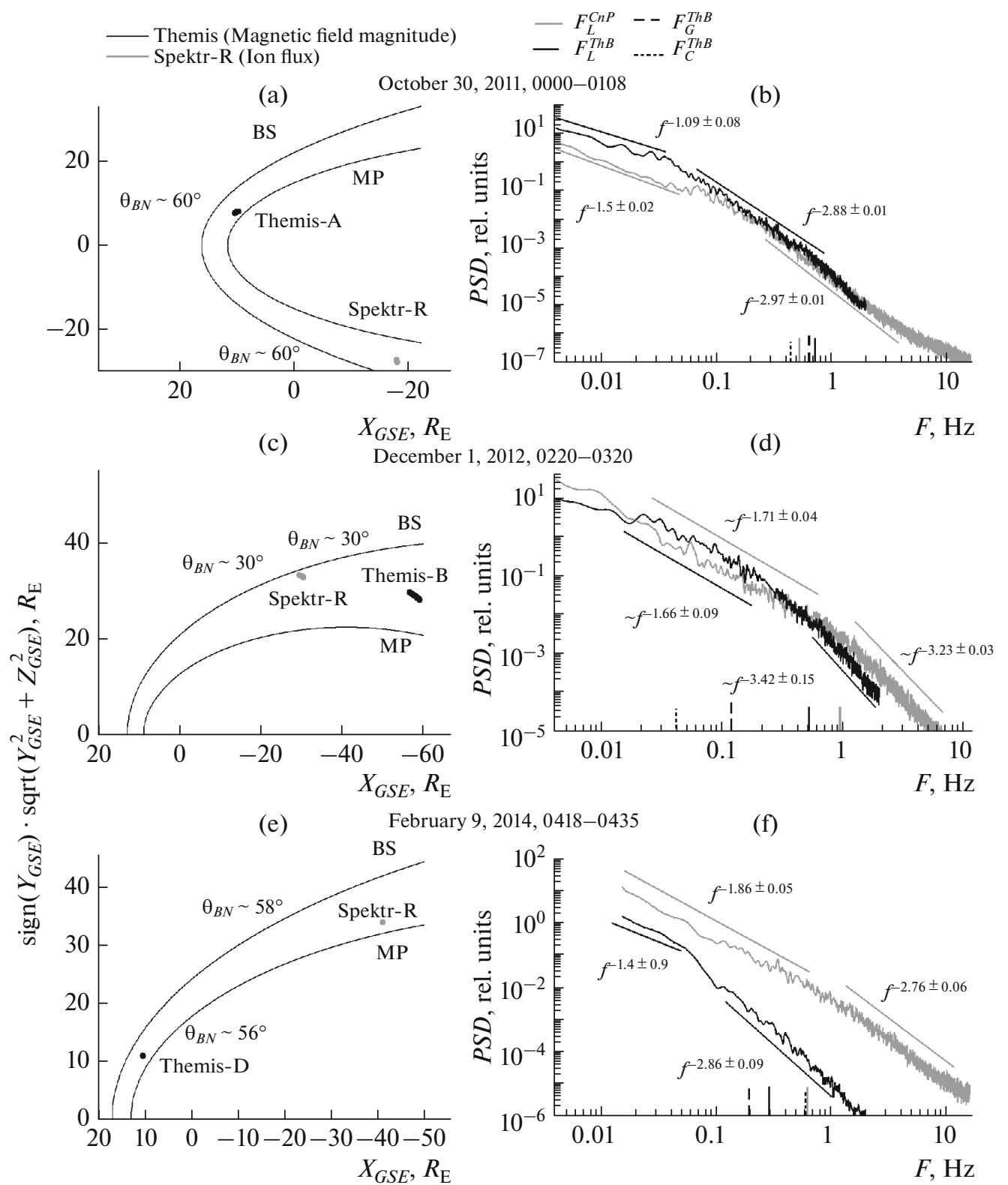

(g)

(h)
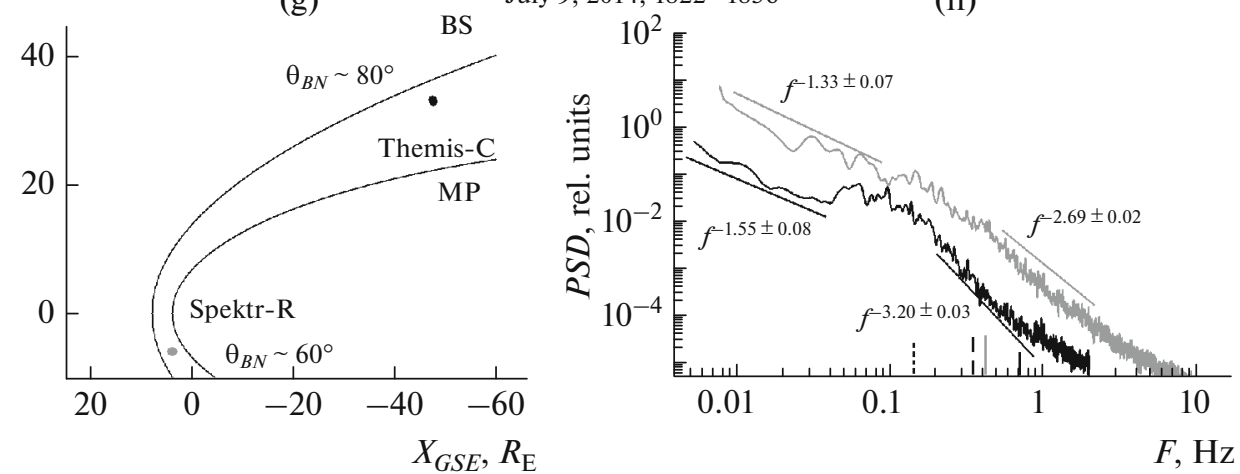

Fig. 2. Left: spacecraft location. Right: spectra of fluctuations of the ion flux (gray line) and magnetic-field magnitude (black line) for the four considered events. 
Table 1. Characteristics of the spectra of ion flux fluctuations by measurements of the Spektr-R satellite and the magneticfield magnitude by measurements of the Themis satellite, the satellite locations, and the value of the angle $\theta_{B N}$ for the considered events

\begin{tabular}{c|l|l|c|c|c}
\hline \multicolumn{1}{c|}{ Event } & Satellite & \multicolumn{1}{c|}{ Location } & Angle $\theta_{B N},{ }^{\circ}$ & $\begin{array}{c}\text { Spectrum slope } \\
\text { on MHD scales }\end{array}$ & $\begin{array}{c}\text { Spectrum slope } \\
\text { on kinetic scales }\end{array}$ \\
\hline October 30, 2011 & Themis & MP, s/s & 60 & $-1.09 \pm 0.08$ & $-2.88 \pm 0.01$ \\
& Spektr-R & Center, f & 60 & $-1.50 \pm 0.02$ & $-2.97 \pm 0.08$ \\
\hline December 1, 2012 & Themis & Center, f & 30 & $-1.66 \pm 0.09$ & $-3.42 \pm 0.15$ \\
& Spektr-R & BS, f & 30 & $-1.71 \pm 0.04$ & $-3.23 \pm 0.03$ \\
\hline February 9, 2014 & Themis & MP, s/s & 56 & $-1.4 \pm 0.9$ & $-2.86 \pm 0.09$ \\
& Spektr-R & MP, f & 58 & $-1.87 \pm 0.05$ & $-2.76 \pm 0.06$ \\
\hline July 9, 2014 & Themis & Center, f & 80 & $-1.55 \pm 0.08$ & $-3.20 \pm 0.03$ \\
& Spektr-R & Center, s/s & 60 & $-1.33 \pm 0.07$ & $-2.69 \pm 0.02$ \\
\hline
\end{tabular}

relative positions of satellites shows that three events involving one of the satellites in the subsolar region (events 1, 3, and 4) are observed behind the quasi perpendicular BS and are characterized by a noticeable deviation of the spectra from the Kolmogorov scaling on the MHD scales. At the same time, even near the subsolar region, the spectra in two of the three cases differ from the spectra with an index of -1 , which was noted in the statistical study (Huang et al., 2017). Since the authors of this work considered not compression fluctuations but fluctuations of the Alfvén component, the resulting difference may indicate a difference in the properties of the compressible and incompressible components of fluctuations near the BS. A statistical analysis by Rakhmanova et al. (2018) showed the difference between the spectra of ion-flux fluctuations from the Kolmogorov type only in the regions near the BS, whereas the spectra had slopes close to $-5 / 3$ on the MHD scales in the middle of the MSH and near the magnetopause. In this work, deviations from the Kolmogorov type of spectra in the subsolar region are observed both in the middle of the MSH (event on July 9, 2014) and near the magnetopause (events on October 30, 2011, and February 9, 2014). The observed difference from the results of the statistical study is most likely due to the fact that most of those considered earlier (Rakhmanova et al., 2018) data that referred to the flank MSH.

In the flank areas, all events beyond the quasi perpendicular BS refer to the middle of the MSH or they are near the magnetopause. These events are characterized by spectra close to Kolmogorov spectra at MHD scales. This indicates that turbulence develops as the plasma moves from the subsolar region to the flanks, and, as a result, spectra are observed on the flanks far from the BS. They are close to the predictions of the theory of developed turbulence on MHD scales. This result is in good agreement with the results of a statistical study (Rakhmanova et al., 2018).
The events observed in the subsolar region behind the quasi perpendicular BS are characterized by spectral slopes on the kinetic scales characteristic of MSH and SW plasma, from -2.9 to -2.7 . In this case, two of the three events (October 30, 2011 and February 9, 2014) are characterized by the unaltered slope when the plasma moves to the flanks, since the spectral slopes coincide when the errors in their determination are take into account. For the event on July 9, 2014, there is a noticeable steepening of the spectrum on the kinetic scales during plasma propagation in the MSH: the slope changes from $-2.69 \pm 0.02$ to $-3.20 \pm 0.03$. However, the corresponding spectrum in Fig. 2h demonstrates the presence of a broad peak at a frequency close to the proton cyclotron frequency, which indicates the presence of local wave phenomena that can lead to additional energy dissipation in this region. Thus, in the absence of local sources of additional energy dissipation, the turbulent cascade on the kinetic scales is not subject to change when the plasma moves behind the quasi perpendicular BS from the subsolar region to the flank.

One of the four events shown in Figs. 2c and 2d refers to the MSH behind the quasi parallel BS. This event was considered in more detail by Rakhmanova et al. (2020); however, in this case, it is interesting to compare the properties of the fluctuation spectra behind the quasi parallel and quasi perpendicular BS. As can be seen, both satellites were in the flank area of the MSH: one was near the BS and the other was further downstream in the middle of the MSH. Both spectra have a Kolmogorov form on MHD scales and a higher slope value on kinetic scales (as compared to those observed on average for $\mathrm{MSH}$ and SW) $-3.42 \pm$ 0.15 near the BS and $-3.23 \pm 0.03$ in the middle of the MSH. Thus, under quiet conditions in the SW behind the quasi parallel BS, no differences are observed on the flanks of the MSH between the spectra on the MHD scale and the predictions of the theories of developed turbulence. Wherein, a significant steepening of the spectra is observed on kinetic scale, which is 
probably indicative of an increased rate of energy dissipation caused by processes involved in the local plasma volume, and the dissipation rate is somewhat higher near the BS than far from it. This result corresponds to the earlier conclusions based on statistics (Rakhmanova et al., 2020).

\section{DISCUSSION AND CONCLUSIONS}

The analysis of four events of the observation of compressible turbulent fluctuations in $\mathrm{MSH}$ at two distanced points made it possible to reveal the main features of the modification of the turbulent cascade on the BS and its further dynamics under quiet conditions in the incoming SW flow.

(1) Significant deviation of the fluctuation spectra on the MHD scales from the Kolmogorov form and the reconstruction of the Kolmogorov scaling of the spectra when the plasma propagates towards the flanks far from the BS is observed behind the quasi perpendicular BS. In this case, as a rule, the spectral slopes have value greater in magnitude than -1 , which was obtained statistically for Alfven fluctuations in the subsolar region of the MSH. This may indicate a difference in the modification of the compressible and incompressible components of fluctuations on the BS.

(2) Behind the quasi perpendicular BS on kinetic scales, as a rule, spectra are observed with a slope $-(2.7-2.9)$ characteristic of plasma and magnetic field fluctuations in the $\mathrm{SW}$ and $\mathrm{MSH}$, while this value is retained during plasma propagation from the subsolar region to the flanks in the absence of local wave processes, which can lead to increased dissipation and steepening of the spectrum.

(3) Behind the quasi parallel BS in the flank regions, the spectra correspond to Kolmogorov spectra, regardless of the distance to the BS, while the dissipation rates apparently increase on the kinetic scales, which leads to a significant steepening of the spectra at different distances from the BS and the subsolar region.

This study clearly shows for the first time that the characteristics of the turbulent plasma cascade under quiet conditions in the SW behind the quasi perpendicular BS change significantly on the MHD scales while remaining unchanged on the subionic scales. The subsequent selection of intervals with the same BS topology and satellite locations but different conditions of the SW incoming flow is required in order to determine and separate the contributions of the BS topology and SW characteristics to the development of plasma turbulence in the MSH.

\section{ACKNOWLEDGMENTS}

The authors express their gratitude to their colleagues at the Institute of Space Research of the Russian Academy of Sciences, the Lavochkin Scientific and Production Enterprise, and Charles University in Prague (Prague, Czech
Republic) for their assistance in the development, debugging, calibration, and flight control, as well as for the acquisition, transfer, and primary processing of scientific information from the BMSW instrument.

\section{FUNDING}

This study was supported by the Russian Foundation for Basic Research (project no. 19-02-00177-a).

\section{OPEN ACCESS}

This article is licensed under a Creative Commons Attribution 4.0 International License, which permits use, sharing, adaptation, distribution and reproduction in any medium or format, as long as you give appropriate credit to the original author(s) and the source, provide a link to the Creative Commons license, and indicate if changes were made. The images or other third party material in this article are included in the article's Creative Commons license, unless indicated otherwise in a credit line to the material. If material is not included in the article's Creative Commons license and your intended use is not permitted by statutory regulation or exceeds the permitted use, you will need to obtain permission directly from the copyright holder. To view a copy of this license, visit http://creativecommons.org/licenses/by/4.0/.

\section{REFERENCES}

Alexandrova, O., Lacombe, C., and Mangeney, A., Spectra and anisotropy of magnetic fluctuations in the Earth's magnetosheath: Cluster observations, Ann. Geophys., 2008, vol. 26, pp. 3585-3596.

https://doi.org/10.5194/angeo-26-3585-2008

Alexandrova, O., Chen, C.H.K., Sorriso-Valvo, L., Horbury, T.S., and Bale, S.D., Solar wind turbulence and the role of ion instabilities, Space Sci. Rev., 2013, vol. 178, pp. 101-139.

Boldyrev, S. and Perez, J.C., Spectrum of kinetic Alfvén turbulence, Astrophys. J. Lett., 2012, vol. 758, no. 2, id L44.

https://doi.org/10.1088/2041-8205/758/2/L44

Bruno, R. and Carbone, V., The solar wind as a turbulence laboratory, Living Rev. Sol. Phys., 2013, vol. 10, id 2. https://doi.org/10.12942/lrsp-2013-2

Chen, C.H.K., Recent progress in astrophysical plasma turbulence from solar wind observations, J. Plasma Phys., 2016, vol. 82, id 535820602 . https://doi.org/10.1017/S0022377816001124

Chen, C.H.K. and Boldyrev, S., Nature of kinetic scale turbulence in the Earth's magnetosheath, Astrophys. J., 2017, vol. 842, id 122.

https://doi.org/10.3847/1538-4357/aa74e0

Chen, C.H.K., Salem, C.S., Bonnel, J.W., Moze, F.S., and Bale, S.D., Electron density fluctuation spectrum of solar wind turbulence between ion and electron scales, Phys. Rev. Lett., 2012, vol. 109, no. 3, 035001. https://doi.org/10.1103/PhysRevLett.109.035001

Huang, S.Y., Sahraoui, F., Deng, X.H., He, J.S., Yuan, Z.G., Zhou, M., Pang, Y., and Fu, H.S., Kinetic turbulence in the terrestrial magnetosheath: Cluster observations, 
Astrophys. J., 2014, vol. 789, id L28. https://doi.org/10.1088/2041-8205/789/2/L28

Huang, S.Y., Hadid, L.Z., Sahraoui, F., Yuan, Z.G., and Deng, X.H., On the existence of the Kolmogorov inertial range in the terrestrial magnetosheath turbulence, Astrophys. J. Lett., 2017, vol. 836, no. 1, id L10. https://doi.org/10.3847/2041-8213/836/1/L10

Li, H., Jiang, W., Wang, C., Verscharen, D., Zeng, C., Russel, C.T., Giles, B., and Burch, J.L., Evolution of the Earth's magnetosheath turbulence: A statistical study based on MMS observations, Astrophys. J., 2020, vol. 898, id L43. https://doi.org/10.3847/2041-8213/aba531

Rakhmanova, L., Riazantseva, M., and Zastenker, G., Plasma fluctuations at the flanks of the Earth's magnetosheath at ion kinetic scales, Ann. Geophys., 2016, vol. 34, pp. 1011-1018.

Rakhmanova, L.S., Riazantseva, M.O., Zastenker, G.N., and Verigin, M.I., Effect of the magnetopause and bow shock on characteristics of plasma turbulence in the Earth's magnetosheath, Geomagn. Aeron. (Engl. Transl.), 2018a, vol. 58, no. 6, pp. 718-727.

Rakhmanova, L., Riazantseva, M., Zastenker, G., and Verigin, M., Kinetic scale ion flux fluctuations behind the quasi-parallel and quasi-perpendicular bow shock, J. Geophys. Res.: Space Phys., 2018b, vol. 123, pp. 5300-5314. https://doi.org/10.1029/2018JA025179

Rakhmanova, L.S., Riazantseva, M.O., Zastenker, G.N., Yermolaev, Yu.I., Lodkina, I.G., and Chesalin, L.S., Turbulent cascade in the magnetosheath affected by the solar wind's plasma turbulence, Cosmic Res., 2019, vol. 57, no. 6, pp. 443-450.

Rakhmanova, L., Riazantseva, M., Zastenker, G., Yermolaev, Y., and Lodkina, I., Dynamics of plasma turbulence at Earth's bow shock and through the magnetosheath, Astrophys. J., 2020, vol. 901, id 30. https://doi.org/10.3847/1538-4357/abae00

Rakhmanova, L., Riazantseva, M., and Zastenker, G., Plasma and magnetic field turbulence in the Earth's magnetosheath at ion scales, Front. Astron. Space Sci., 2021, vol. 7, id 616635 .

https://doi.org/10.3389/fspas.2020.616635
Riazantseva, M.O., Budaev, V.P., Zelenyi, L.M., Zastenker, G.N., Pavlos, G.P., Šafránková, J., Němeček, Z., Přech, L., and Němec, F., Dynamic properties of small scale solar wind plasma fluctuations, Philos. Trans. $R$. Soc., A, 2015, vol. 373.

https://doi.org/10.1098/rsta.2014.0146

Roberts, O.W., Narita, Y., Li, X., Escoubet, C.P., and Laakso, H., Multipoint analysis of compressive fluctuations in the fast and slow solar wind, J. Geophys. Res.: Space Phys., 2017, vol. 122, pp. 6940-6963. https://doi.org/10.1002/2016JA023552

Šafránková, J., Němeček, Z., Přech, L., et al., Fast Solar Wind Monitor (BMSW): Description and first results, Space Sci. Rev., 2013, vol. 175, pp. 165-182.

Šafránková, J., Němeček, Z., Němec, F., Přech, L., Chen, C.H.K., and Zastenker, G.N., Power spectral density of fluctuations of bulk and thermal speeds in the solar wind, Astrophys. J., 2016, vol. 825, id 121. https://doi.org/10.3847/0004-637X/825/2/121

Sahraoui, F., Hadid, L., and Huang, S., Magnetohydrodynamic and kinetic scale turbulence in the near-Earth space plasmas: A (short) biased review, Rev. Mod. Phys., 2020, vol. 4, id 4.

https://doi.org/10.1007/s41614-020-0040-2

Shevyrev, N.N. and Zastenker, G.N., Some features of the plasma flow in the magnetosheath behind quasi-parallel and quasi-perpendicular bow shocks, Planet. Space Sci., 2005, vol. 53, pp. 95-102. https://doi.org/10.1016/j.pss.2004.09.033

Spreiter, J.R., Summers, A.L., and Alksne, A.Y., Hydromagnetic flow around the magnetosphere, Planet. Space Sci., 1966, vol. 14, pp. 223-253. https://doi.org/10.1016/0032-0633(66)90124-3

Yermolaev, Yu.I., Nikolaeva, N.S., Lodkina, I.G., and Yermolaev, M.Yu., Catalog of large-scale solar wind phenomena during 1976-2000, Cosmic Res., 2009, vol. 47, no. 2, pp. 81-94.

Zastenker, G.N., Šafránková, J., Němeček, Z., et al., Fast measurements of parameters of the solar wind using the BMSW instrument, Cosmic Res., 2013, vol. 51, no. 2, pp. $78-89$. 